\title{
Rehabilitation in hemato-oncology
}

\author{
Marco Hassler
}

Hemato-oncology is a rapidly evolving discipline, challenging clinicians to stay up-to-date. Progress in treatment is impressive, including solid tumors, i.e. melanoma or lung cancer as well as hematologic malignancies like multiple myeloma.

As a result of these advances, it is interesting to note that public opinion about cancer is changing. For many years, a diagnosis of cancer or leukemia was associated with death, but it is now accepted as a chronic disease by both patients and caregivers.

Another consequence is that clinicians are increasingly confronted not only with aspects of direct antineoplastic treatment decisions and therapies but attendance of side effects, supportive therapies, psychosocial aspects and other issues like the cancer survivor's needs.

Advances in medical treatment also reflects progress in supportive treatment. Thus, based on knowledge from medical textbooks in the 1990s, physicians had to recommend that their oncologic patients rest and avoid physical exertion and challenges. The first papers with positive effects of exercise in cancer patients was published as a thesis in 1983 and has expanded to guidelines with specific recommendations for endurance and resistance exercise training.

Some clinical situations need special considerations. A very interesting aspect is the handling of exercise in patients with bone metastases, which is a challenge for radiologists, orthopedists and clinicians, especially in the outpatient setting without the possibility of a specialist's consultation. Winfried Habelsberger summarizes challenges and effects in

M. Hassler, MD (ه)

Special Hospital for Oncological Rehabilitation "Der

Sonnberghof", 7202 Bad Sauerbrunn, Austria

m.hassler@dersonnberghof.at patients with bone metastases taking into consideration physical training and its supervision [1].

A further very interesting and common symptom is chemotherapy-induced peripheral neuropathy. As medical treatment very often remains unsatisfactory, up to $50 \%$ of patients report symptoms of polyneuropathy in the inpatient oncological rehabilitation clinic setting (Vogt, 2010, www.argekrebsnw. de/Foerderung-von-Forschungsprojekten) and $80 \%$ of these patients ultimately expect occupational impairments. Thomas Licht describes treatment options for chemotherapy-induced peripheral neuropathy [2].

Oncological rehabilitation, mainly in an inpatient setting, is applied for in Austria after specific cancer treatment, and, as described above, we are able to estimate the aims and symptoms the patients will report, sometimes as consequences of the disease and sometimes as side effects of treatment. As Richard Crevenna describes in his essay, several years ago interventions were implemented before and during treatment to improve the functional status or to minimize an expected loss of function. These interventions include training of the pelvic floor before prostatectomy and respiratory training before lobectomy [3].

These three papers from my esteemed colleagues contribute to a concept of care that constitutes our aim: "the continuum of cancer care" from the diagnosis of a malignant disease, during and even long after antineoplastic treatment until the end of life setting. Thus, I believe that care never ends and there is always something that can be done for a patient!

Conflict of interest M. Hassler declares that he has no competing interests. 


\section{editorial}

\section{References}

1. Habelsberger W. Physical activity and exercise in cancer patients with bone metastases. memo. 2021;14(1). https:// doi.org/10.1007/s12254-020-00664-3.

2. Licht T, Keilani M, Crevenna R. Chemotherapy-induced peripheral neuropathy (CIPN). memo. 2021;14(1). https:// doi.org/10.1007/s12254-021-00688-3.

3. Crevenna R, Palma S, Licht T. Cancer prehabilitation-a short-review. memo. 2021;14(1). https://doi.org/10.1007/ s12254-021-00686-5.
Publisher's Note Springer Nature remains neutral with regard to jurisdictional claims in published maps and institutional affiliations.

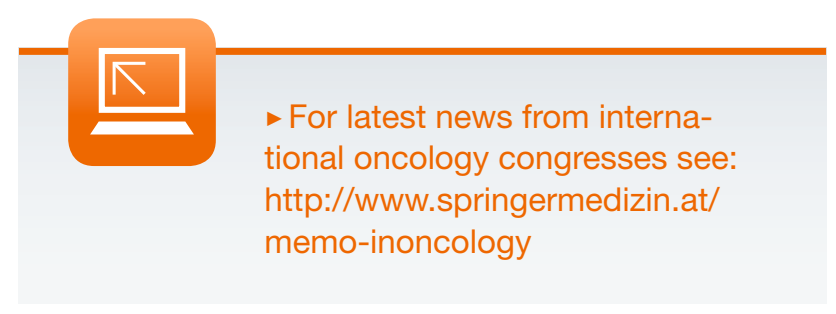

